\title{
BMJ Open Randomised phase II trial of olaparib, chemotherapy or olaparib and cediranib in patients with platinum-resistant ovarian cancer (OCTOVA): a study protocol
}

\author{
Anita Mansouri (D) , ${ }^{1}$ Naomi McGregor, ${ }^{2}$ Rachel Dunn, ${ }^{2}$ Sam Dobbie, ${ }^{2}$ \\ Jane Holmes, ${ }^{1}$ Linda Collins, ${ }^{2}$ Shibani Nicum ${ }^{3}$
}

To cite: Mansouri A,

McGregor N, Dunn R, et al. Randomised phase II trial of olaparib, chemotherapy or olaparib and cediranib in patients with platinum-resistant ovarian cancer (OCTOVA): a study protocol. BMJ Open 2021;11:e041463. doi:10.1136/ bmjopen-2020-041463

- Prepublication history and additional material for this paper is available online. To view these files, please visit the journal online (http://dx.doi.org/10. 1136/bmjopen-2020-041463).

Received 11 June 2020

Revised 02 November 2020

Accepted 15 November 2020

Check for updates

(C) Author(s) (or their employer(s)) 2021. Re-use permitted under CC BY-NC. No commercial re-use. See rights and permissions. Published by BMJ.

${ }^{1}$ Oxford Clinical Trials Research Unit, Centre for Statistics in Medicine, Nuffield Department of Orthopaedics, Rheumatology and Musculoskeletal Sciences, University of Oxford, Oxford, UK ${ }^{2}$ Oncology Clinical Trials Office (ОСТ0), Department of Oncology, University of 0 xford, Oxford, UK

${ }^{3} 0 x f o r d$ Cancer and

Haematology Centre, Oxford University Hospitals NHS Foundation Trust, Oxford, UK

Correspondence to

Dr Shibani Nicum;

snicum@nhs.net

\section{ABSTRACT}

Introduction Patients relapsing within 12 months of platinum-based chemotherapy usually have a poorer response to subsequent treatments. To date, extensive research into the mechanism of resistance to platinum agents in the treatment of ovarian cancer has not resulted in improved responses or longer survival. Further experimental work and clinical trials with novel agents are therefore justified to address this unmet need. Patients with ovarian, fallopian tube or primary peritoneal cancer that has relapsed within 12 months of platinum-based chemotherapy will be randomised with stratification for BReast CAncer gene (BRCA) status, prior poly (ADP-ribose) polymerase (PARP) exposure and prior antiangiogenic therapy into weekly paclitaxel (chemotherapy), olaparib or the combination of cediranib and olaparib. They will be followed until disease progression or unacceptable toxicity develops. Our trial design permits two investigations. We will compare the efficacy and tolerability of single-agent olaparib with weekly paclitaxel. We will also compare the efficacy and tolerability of olaparib with the combination of olaparib and cediranib. The required sample size of 138 participants (46 per arm) was calculated using a $20 \%$ one-sided type I error, $80 \%$ power and $15 \%$ dropout rate. Recruitment will last 34 months with a follow-up of 18 months.

Methods and analysis

Ethics and dissemination This study will be conducted under a UK Medicines and Healthcare Products Regulatory Agency Clinical Trials Authorisation. Approval to conduct the study was obtained from the responsible authority before beginning the study. The sponsor will retain ownership of all data arising from the trial. We aim to publish this research in a specialist peer-reviewed scientific journal on study completion. EudraCT number: 2016-000559-28, ethics reference number: 16/L0/2150.

Trial registration number ISRCTN: ISRCTN14784018, clinicaltrials.gov: NCT03117933; Pre-results.

\section{INTRODUCTION}

Background and rationale

Ovarian cancer is the sixth most common malignancy in the UK and the most common
Strengths and limitations of this study

- OCTOVA includes current standard of care treatment and allows participants in the standard of care treatment arm to crossover to receive Investigational Medicinal Product (IMP).

- This trial contains a large number of patients with BRCA in comparison to other similar studies.

- This is the first trial we know of to include poly (ADPribose) polymerase (PARPi) retreatment data.

- Limitations include initially requiring participants with a BReast CAncer gene (BRCA) mutation that affected recruitment rates.

- This trial is unblinded, which may have influenced a minority of treatment decisions.

cause of death from gynaecologic malignancy. $^{12}$

Seventy per cent of patients present with advanced ovarian cancer, at stage III or IV, and although they initially respond well to platinum-based chemotherapy, the majority will relapse. ${ }^{3}$ Patients relapsing within 12 months of platinum therapy have a poorer response to subsequent treatments than those relapsing later, and as a result have a decreased overall survival. ${ }^{4}$ In order to address this unmet need, experimental work and clinical trials with novel agents have focused on improving survival by maximising tumour cell kill and also reducing toxicity by providing an efficacious alternative to chemotherapy.

Molecular targeted therapy using poly (ADP-ribose) polymerase (PARP) inhibitors has been shown to be effective in the treatment of both frontline and relapsed ovarian cancer. PARP inhibitors exploit biological pathways within tumour cells that differ from those in normal cells. ${ }^{5-7}$ It has been shown that olaparib (AZD2281) as an orally PARP 
inhibitor has a clinical efficacy in patients with newly ${ }^{8}$ diag- $^{-}$ nosed and relapsed ovarian cancer with BReast CAncer gene 1 (BRCA1) or BRCA2 deficiency ${ }^{9}{ }^{10}$ and in those who are BRCA wild type. ${ }^{11}$ Olaparib has the potential to provide an efficacious and less toxic treatment option. It is indicated as monotherapy for the maintenance treatment of women with newly diagnosed and relapsed, platinumsensitive high-grade serous epithelial ovarian, fallopian tube or primary peritoneal cancer, but it is not licensed in the treatment of women with platinum-resistant ovarian cancer.

An essential step in tumour growth and metastasis is the process of new blood vessel formation, angiogenesis. The inhibition of angiogenesis has therefore emerged as a key strategy for treating cancer, often by inhibiting vascular endothelial growth factor (VEGF), which stimulates blood vessel formation. Anti-VEGF agents such as bevacizumab and cediranib, used as single agents or in combination with chemotherapy or PARP inhibitors, have shown improved progression-free survival in women with relapsed ovarian cancer. ${ }^{12-15}$

Cediranib combined with olaparib has demonstrated significant clinical activity in treating patients of any BRCA status who have platinum-sensitive relapsed ovarian cancer. ${ }^{13}$ The combination of cediranib and olaparib significantly extended progression-free survival and overall response rates, compared with olaparib in platinum-sensitive ovarian cancer and therefore warrants further investigation in the platinum-resistant setting in both BRCA wild type and mutated disease. The observed synergy between olaparib and cediranib maybe as a result of hypoxia related to the antiangiogenic effect of cediranib or the inhibition of Platelet Derived Growth Factor (PDGF) signalling by cediranib, which results in the down-regulation of homologous recombination (DNA repair) genes, increasing the susceptibility to PARP inhibition. ${ }^{1316}$

Paclitaxel is an important agent used in the treatment of early stage and relapsed ovarian cancer. Preclinical studies including in ovarian cancer models have demonstrated that fractionated paclitaxel dosing is associated with increased antiangiogenic and proapoptotic effects as well as reduced leucopenia. ${ }^{17-21}$ In the platinum-resistant setting, the majority of patients, irrespective of BRCA status, will receive weekly paclitaxel. Women who relapse within 12 months of prior platinum therapy have a degree of platinum resistance, and therefore it is acceptable to consider the use of platinum sparing options, such as weekly Taxol or Caelyx, in this group. Caelyx is often used earlier in the treatment pathway in combination with carboplatin, and therefore we have chosen weekly taxol as our comparator arm. A retrospective analysis demonstrated that weekly Taxol had similar efficacy in sporadic and BRCA-mutated relapsed patients with ovarian cancer. ${ }^{22}$ The study, conducted in four cancer centres, analysed response and Progression Free Survival (PFS) following paclitaxel (3-weekly/weekly) monotherapy in BRCA-mutated relapsed patients with ovarian cancer.
There were 26 patients, 15 platinum-sensitive (58\%) and 11 platinum-resistant $(42 \%)$ patients. The clinical benefit rate (complete or partial response or stable disease) was $36 \%$, with a median PFS of 21 weeks, ${ }^{22}$ which is consistent with the PFS of 4.7-5.3 months in the SaPPrOC study, in which patients with unknown BRCA status and platinumresistant ovarian cancer received weekly Taxol. ${ }^{23}$

\section{Objectives}

The primary objective is to evaluate the efficacy of olaparib compared with weekly paclitaxel or the combination of olaparib and cediranib in participants with ovarian, fallopian tube or primary peritoneal cancer who have relapsed within 12 months of previous platinum therapy.

Secondary objectives are to evaluate the safety and tolerability of the combination of olaparib and cediranib, overall survival, objective response rate and quality of life.

\section{Trial design}

This trial is a multicentre, randomised phase II trial. The trial has recruited 139 patients over 34 months. Participants were randomised with stratification for BRCA status, prior PARP exposure and prior antiangiogenic therapy into weekly paclitaxel (chemotherapy), olaparib or the combination of cediranib and olaparib. Drug doses are based on those standardly used for paclitaxel and the recommended monotherapy tablet dose for olaparib and cediranib. Participants receive trial medication until disease progression or unacceptable toxicity develops. Those who progress on weekly paclitaxel are permitted to cross over and receive single-agent olaparib therapy. Trial participants are permitted to remain on olaparib for as long as they are clinically benefiting. Survival updates are required for up to 18 months postprogression. Participants who are still receiving treatment at 18 months will remain in the trial but only safety data will be collected. Participants in the trial may continue to receive the Investigational Medicinal Product (IMP) they were randomised to (or in paclitaxel-only patients, olaparib, if they have progressed) until disease progression in the absence of toxicity.

The Randomised phase II trial of olaparib, chemotherapy or olaparib and cediranib in patients with platinum-resistant ovarian cancer (OCTOVA) trial design permits two separate investigations: the comparison of the efficacy and tolerability of single-agent olaparib with weekly paclitaxel and the efficacy and tolerability of olaparib with the combination of olaparib and cediranib. Based on the previous studies, we expect olaparib to be more efficacious and tolerable than chemotherapy. Participant-reported quality of life data will also form an important part of this study.

The first participant was randomised on 30 May 2017 and the final participant randomised on 10 Jan 2020. 


\section{METHODS}

\section{Study setting}

The trial included the following UK recruiting centres: Beatson Cancer Centre, Belfast City Hospital, The Christie, Churchill Hospital, Clatterbridge Cancer Centre, Hammersmith Hospital, Mount Vernon Cancer Centre, Nottingham City Hospital, Royal Marsden Hospital Chelsea and Sutton, Royal Surrey County Hospital, Royal United Hospital, St Bartholomew's Hospital, University College London Hospitals, Velindre Cancer Centre.

\section{Eligibility criteria}

Female patients aged $\geq 16$ years with relapsed epithelial ovarian, primary peritoneal or fallopian tube cancer that has relapsed within 12 months of previous platinumbased therapy (which does not have to be the most recent chemotherapy) will be eligible for this trial, provided they meet the following criteria.

Patients have received prior PARP inhibitor and/or prior antiangiogenic therapy, but at least 6 months must have passed (6 weeks for bevacizumab) since treatment.

Patients should have a measurable disease confirmed by RECIST V.1.1 in the past 4 weeks.

Patients must have haemoglobin $\geq 9.0 \mathrm{~g} / \mathrm{dL}$, have received no blood transfusions in the 28 days before randomisation and have an Eastern Cooperative Oncology Group performance status of 0-2. They must have normal organ and bone marrow function measured within 14 days before study treatment begins, defined by absolute neutrophil count $\geq 1.5 \times 109 / \mathrm{L}$; platelet count $>100 \times 109 / \mathrm{L}$; total bilirubin $\leq 1.5 \times$ institutional upper limit of normal (ULN); serum creatinine $\leq 1.5 \times$ ULN or calculated creatinine clearance $>50 \mathrm{~mL} / \mathrm{min}$ calculated using Cockroft-Gault, Jelliffe or Wright and urine dipstick for proteinuria $<2+$. If urine dipstick is $\geq 2+$ on two occasions more than 1 week apart, then a 24 hours urine must demonstrate $\leq 1 \mathrm{~g}$ of protein in 24 hours or protein/creatinine ratio $<1.5$.

Patients must have sufficient archival tissue confirming histological diagnosis available and a life expectancy of more than 12 weeks for disease-related mortality. They must be able to swallow, retain oral medications and comply with the protocol for the study duration, including undergoing treatment and scheduled visits and examinations, including follow-up.

Patients will not be eligible if they have ever received single-agent weekly paclitaxel for relapsed disease, been treated with any other investigational agent or systemic chemotherapy or participated in another interventional clinical trial within the 28 days before enrolment. They must not have received a radiotherapy dose within 2 weeks before the study treatment begins.

Patients with poorly controlled hypertension are ineligible, defined as persistently elevated blood pressure $>150 / 100 \mathrm{~mm} \mathrm{Hg}$, systolic, diastolic or both, despite antihypertensive medication. Patients with a history of inflammatory bowel disease or cerebrovascular accident (including transient ischaemic attacks) within the last
12 months are ineligible. Patients with gastrointestinal impairment that could affect their ability to take or absorb oral medicines, including subacute or complete bowel obstruction, evidence of severe or uncontrolled cardiac disease, active bleeding or bleeding diathesis defined as significant haemorrhage $(>30 \mathrm{~mL}$ bleeding/episode in previous 3 months) or haemoptysis ( $>5 \mathrm{~mL}$ fresh blood in previous 4 weeks), are ineligible.

Patients known to be serologically positive for hepatitis B, hepatitis C, or HIV or immunocompromised (eg, patients taking immunosuppressive drugs) are ineligible for the trial. Those who have started a stable dose of bisphosphonates for bone metastases less than 4 weeks before treatment with the study drug and those with concomitant use of known CYP3A4 inhibitors or potent inducers of CYP3A4 cannot participate. Patients with persistent toxicities (adverse event of National Cancer Institute (NCI) Common Terminology Criteria for Adverse Events (CTCAE) V.4.03 grade 2 or more) caused by previous cancer therapy (except alopecia), myelodysplastic syndrome, acute myeloid leukaemia and symptomatic, untreated, uncontrolled brain or meningeal metastases or tumours are ineligible.

Patients who have had major surgery within 14 days of starting the study treatment or have not recovered from any effects of any major surgery are ineligible for the trial. Patients considered a poor medical risk due to a serious, uncontrolled medical disorder, non-malignant systemic disease or active, uncontrolled infection and patients with left ventricular ejection fraction less than the institutional lower limit of normal or resting ECG with corrected QT interval (QTc) $>470 \mathrm{~ms}$ or family history of long QT syndrome must be excluded.

Patients with known treatment-limiting hypersensitivity to cediranib, olaparib, paclitaxel or any of its excipients or any other psychological, social or medical condition, physical examination finding or laboratory abnormality that the investigator considers would make the patient a poor trial candidate or could interfere with protocol compliance or the interpretation of trial results must be excluded. Patients with any other active malignancy that requires treatment or whose prognosis will prevent readout from trial endpoints, except adequately treated cone-biopsied in situ carcinoma of the cervix uteri and non-melanoma skin lesions must be excluded. Pregnant and breastfeeding women are ineligible, as are women of childbearing potential unless they use effective methods of contraception during the trial and for 6 months after stopping treatment.

Participants must sign to confirm their informed consent (see online supplemental file 1) before any study-specific procedures and be capable of cooperating with the protocol. Patients with psychiatric disorders that prohibit obtaining informed consent are not eligible.

\section{Interventions}

Participants receive paclitaxel $\left(80 \mathrm{mg} / \mathrm{m}^{2}\right.$ weekly administered over 1 hour by intravenous infusion on days 1,8 and 
15 , every 28 days) or olaparib ( $300 \mathrm{mg}$ two times per day as tablets orally) or both cediranib $(20 \mathrm{mg}$ /day as tablets orally) and olaparib (300 mg two times per day as tablets orally).

Participants receive treatment until disease progression, unacceptable toxicity or the participant asks to stop treatment. Participants who progress on weekly paclitaxel will be permitted to cross over and receive single-agent olaparib therapy. They can continue to receive olaparib beyond RECIST V.1.1 progression for as long as they derive clinical benefit and do not need to initiate another therapy. Participants may continue until the next line of therapy is started.

Any toxicity observed during the study will be managed by interruption and/or dose adjustment. A dose reduction will be considered for participants experiencing higher grade adverse events or several adverse events. If an adverse event occurs that is potentially related to both olaparib and cediranib, both doses will be modified. If the adverse event is only attributable to one trial drug, then this dose will be reduced and the other trial drug may continue at the same dose. Participants will remain on the reduced dose once a dose reduction of a trial drug has been made. If a trial drug is interrupted for more than 2 weeks, that participant's continuation in the trial will be discussed with the chief investigator.

Participants on paclitaxel will start treatment at a dose of $80 \mathrm{mg} / \mathrm{m}^{2}$. There are two possible dose reduction levels $\left(70 \mathrm{mg} / \mathrm{m}^{2}\right.$ and $\left.60 \mathrm{mg} / \mathrm{m}^{2}\right)$. Before day 1 of every 4 -week chemotherapy cycle, the neutrophil count, platelet count and haemoglobin level must be more than $5 \times 10^{9} / \mathrm{L}$, $100 \times 10^{9} / \mathrm{L}$ and $90 \mathrm{~g} / \mathrm{L}$, respectively. Serum creatinine must be less than $1.5 \times$ ULN, alkaline phosphatase less than $5 \times$ ULN, bilirubin equal or less than $1.5 \times$ ULN and aspartate aminotransferase or alanine aminotransferase equal or less than $2.5 \times$ ULN. Any participant treated at dose reduction level 1 whose bloods fail to meet these criteria on day 1 and does not recover within 7 days will be moved to dose reduction level $2\left(60 \mathrm{mg} / \mathrm{m}^{2}\right)$. Participants at dose reduction level 2 who experience dose-limiting toxicity will be taken off the study. If a dose reduction in paclitaxel has occurred, the dose will not be re-escalated at subsequent cycles.

Doses of olaparib can be reduced to 250, 200 or $150 \mathrm{mg}$ two times per day. Treatment must be interrupted if any NCI-CTCAE V.4.03 grade 3 or 4 adverse event occurs that the investigator considers to be related to the olaparib. Treatment will resume only once the participant recovers completely or the toxicity reverts to NCI-CTCAE V.4.03 grade 1 or less. If toxicity reoccurs following rechallenge with olaparib and further dose interruptions are considered inadequate for managing toxicity, then the participant should be considered for dose reduction or must permanently discontinue treatment with olaparib.

Cediranib doses can be reduced to $20 \mathrm{mg}$ /day for 5 days followed by 2 days off treatment or to $15 \mathrm{mg} /$ day. Any participant who develops any of the following should not receive any further treatment with cediranib: any gastrointestinal perforation or wound dehiscence, arterial thromboembolic events, grade 4 haemorrhagic event (requiring blood transfusion or major non-elective intervention), grade 4 hypertension (hypertensive crisis), nephrotic syndrome or grade 4 proteinuria or reversible posterior leukoencephalopathy syndrome (confirmed by imaging). If a participant develops a second episode of an event at NCI-CTCAE grade 3, then the investigator should not restart cediranib without first discussing the risks and benefits with the chief investigator.

\section{Outcomes}

The primary objective is progression-free survival, measured as time from date of randomisation to RECISTdefined progression or death from any cause (whichever is first).

Adverse events using NCI-CTCAE V.4.03 analysis will evaluate the secondary objective of safety and tolerability of the combination of olaparib and cediranib. Other secondary outcomes are overall survival, objective response rate based on RECIST V.1.1 and GCIG CA125 criteria and quality of life based on EQ5D, EORTC-QLQ C30 and OV28.

\section{Participant timeline}

A summary schedule of events and collection of data at each time point are provided in table 1 .

\section{Sample size}

The required sample size of 138 participants (46 per arm) was calculated using a $20 \%$ one-sided type I error, $80 \%$ power and $15 \%$ dropout rate. Recruitment will last 34 months and follow-up 18 months. We plan to make two pairs of primary endpoint (progression-free survival) comparisons. For the paclitaxel versus olaparib comparison, this sample size with an expected 87 events has $80 \%$ power to detect a HR of 1.44 , which translates to an expected median progression-free survival of 5 months on paclitaxel and 7.2 months on olaparib. For the olaparib and cediranib versus olaparib comparison, this sample size with an expected 77 events has $86 \%$ power to detect a HR of 0.64 , which translates to a median progression-free survival of 7.2 months on olaparib and 11.25 months on olaparib and cediranib.

\section{Recruitment}

The principal investigators and their teams at participating sites identified participants and confirmed eligibility. If there was any doubt about eligibility, the sites were required to consult the chief investigator before recruiting the participant. Participants who did not meet inclusion/exclusion criteria could be rescreened at a later date.

Screening information of all screened participants including any that were subsequently excluded were recorded on a screening log. The original screening log is retained on site and a copy sent to the OCTOVA Trial Office. 


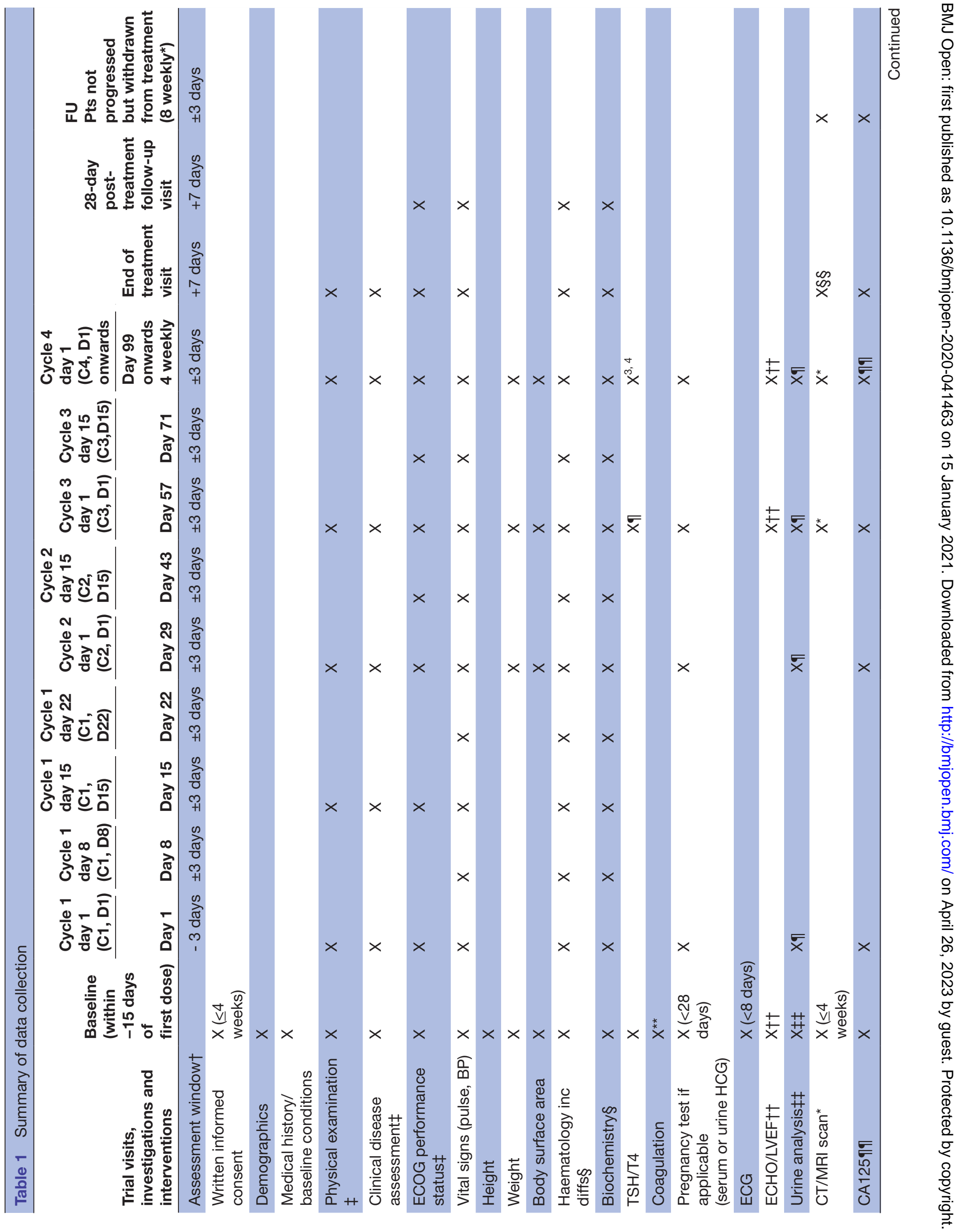




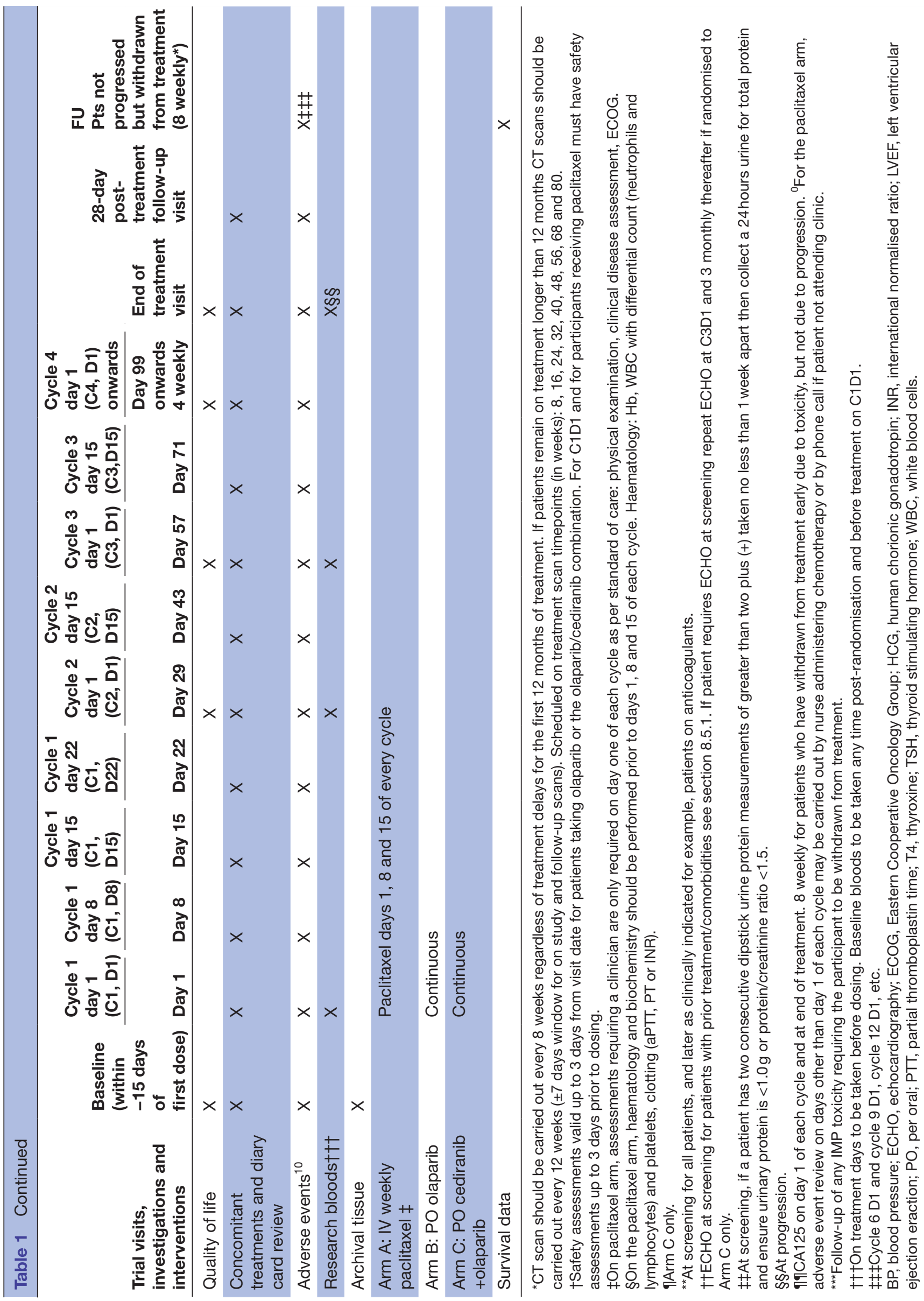

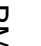

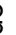

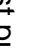

the 


\section{Assignment of interventions}

This trial is an open-label study. Participants were randomised with stratification for BRCA status (BRCA mutant vs BRCA wildtype), prior PARP exposure, and prior anti-angiogenic therapy. If BRCA status is unknown, the participant was stratified as BRCA wildtype.

Site staff completed the trial randomisation form and emailed it to the OCTOVA Trial Office with a suitably anonymised copy of the histology to confirm the participants' eligibility and BRCA report if available.

\section{Data collection and management}

OpenClinica, a validated online clinical database is used for collection of the clinical data. Sites are provided with instructions and a video link for training purposes. The participants are identified by a unique trial-specific number and/or code in each database.

The Oxford Clinical Trial Office monitors the trial, evaluating the data for compliance with the protocol, completeness and accuracy. The case report data are validated using appropriate set criteria and range and verification checks. The study sites must resolve all data queries in a timely manner. All queries are referred to the study site for resolution.

\section{Early participant withdrawal}

A participant can withdraw early from treatment due to unacceptable toxicity, adverse events or serious adverse events requiring discontinuation, loss to follow-up, significant protocol deviation, inability to comply with trial procedures, a clinical decision (other than disease progression), the participant's decision or pregnancy. Withdrawn participants are followed up for disease progression. The investigators are responsible for following up any serious adverse events experienced by withdrawn participants until resolution.

\section{Interim analysis}

A safety check was carried out after the first 15 patients reached the primary endpoint, and then at least once every 6 months. Safety data are reviewed by an internal safety monitoring committee including an oncologist independent from the trial, the trial statistician and a member of the trials' office.

\section{Statistical methods}

The intention-to-treat population will be used for all final analyses. This population will include all participants who gave their informed consent and were successfully randomised. Safety analyses will be done on a safety population basis, including all participants who were randomised and received at least one dose of one treatment.

The primary endpoint, progression-free survival, will be reported as a time-to-event variable, defining length of survival in whole days as the time from randomisation until progression or death from any cause (whichever occurs first). Progression-free survival will be analysed when approximately 87 disease progression or death events have occurred in the paclitaxel and olaparib arms, combined. For each pair of comparisons-paclitaxel versus olaparib and olaparib plus cediranib versus olapar$\mathrm{ib}$ - $\mathrm{a}$ Cox regression model adjusting for the stratification factors will be used. HRs with $60 \%$ and $80 \%$ CIs alongside Kaplan-Meier plots will be reported. A significant level of 0.2 will be used to declare statistical significance. Generalised linear modelling will be used to analyse quality of life questionnaires over time.

Objective response rates will be compared using proportions and responses will be graphically represented. An adverse event summary will be provided based on all available participants. Overall survival rate at 12 and 18 months for each group will be reported. ${ }^{24}$ If more than $50 \%$ of participants cross over treatments, we will report this subgroup's overall survival rate at 12 and 18 months.

Missing data will be chased up where possible after consultation with the investigators.

\section{Oversight and monitoring}

An independent early phase trials oversight committee (IEPTOC) covers the role of an independent data and safety monitoring committee. This committee assesses the trial data and monitors trial recruitment, protocol compliance, toxicity and serious adverse events every 6 months during recruitment and annually thereafter. Safety data are reviewed as described above.

The successful conduct and publication of the trial are overseen by the trial management group, which includes the chief investigator, coinvestigators, clinical trial coordinator, trial statistician, an independent oncologist and others as required. The group meets as necessary to discuss trial data and progress.

\section{Harms}

Any toxicity observed during the study is managed by interruption and/or dose adjustment, as described in the intervention section.

Throughout the study, participants are routinely monitored by investigators for clinical and laboratory evidence of adverse events. Adverse events from the time the participant begins treatment until they complete the 28-day follow-up visit must be reported and followed to a satisfactory conclusion. Any reportable drug-related adverse events that are unresolved at the end of the treatment visit should be followed up by the investigator until resolution or stabilisation. All adverse events must be graded according to the NCI-CTCAE V.4.03. Any studydrug-related serious adverse events require expedited reporting and must also be reported in the Case Report Form (CRF) and followed to resolution or stabilisation.

\section{Auditing}

All aspects of the study's conduct may be subjected to internal or external quality assurance audits to ensure compliance with the protocol, good clinical practice requirements and other applicable regulation or standards. The study may also be subjected to a regulatory 
inspection. Audits and inspections can occur at any time during or after the study. Investigators and their host institutions will allow auditors or inspectors direct access to all relevant documents and study facilities and will allocate their and their staff's time to facilitate the audit or inspection visit. Anyone receiving notification of a regulatory inspection that will (or is likely to) involve this trial must inform the trial office without delay.

\section{Patient and public involvement}

Patients were not involved in the design of this study. The Patient Information Sheet (PIS), Patient Information Consent Form (PICF), poster and website were reviewed by patients before submission to the Research Ethics Committee (REC). The IEPTOC includes a consumer representative who will be involved in assessing the trial data and monitoring the trial recruitment, protocol compliance and toxicity.

\section{Ethics and dissemination}

\section{Research ethics approval}

The London-Chelsea REC reviews and approves principal investigators, protocol and all information that is given to eligible participants including patient information sheet and consent form.

This study is being conducted under UK Medicines and Healthcare Products Regulatory Agency Clinical Trials Authorisation. Approval to conduct the study was obtained from the responsible authority before the study began. EudraCT number: 2016-000559-28, ethics reference number: $16 / \mathrm{LO} / 2150$.

The University of Oxford (Clinical Trials and Research Governance Team, Joint Research Office, Block 60, Churchill Hospital, Old Road, Headington, Oxford, OX3 7LE, Email: ctrg@admin.ox.ac.uk) is the sponsor.

\section{Protocol amendments}

The trial opened to recruitment using protocol V.2.0 (04 January 2017). This manuscript is written based on protocol V.6.0 (09 July 2019). Some substantial amendments were:

- Removal of the exclusion criterion 'risk of thrombotic events'.

- Removal of the requirement to fast with olaparib.

- Removal of pharmacokinetic sampling.

- Addition of the requirement to report cases of Hy's law.

- Clarification of cardiac risk requiring an Echocardiography (ECHO) at screening.

- For clarification, paclitaxel arm patients need to see a clinician on day 1 of cycle only.

- Clarification that anthracycline cardiac risk does not include liposomal doxorubicin.

- Clarification of haematology eligibility requirements.

- Clarification safety reporting is from first dose of IMP.

- Clarification of management of Left Ventricular Ejection Fraction (LVEF) changes.

- Clarification of window for assessment visits.
- Reduction of bevacizumab washout from 6 months to 6 weeks.

- Inclusion criterion for haemoglobin reduced from 10 $\mathrm{g} / \mathrm{dL}$ to $9 \mathrm{~g} / \mathrm{dL}$.

- Removal of BRCA mutation requirement.

- Altered definition of platinum resistant to 'relapse within 12 months of platinum treatment'.

- Removal of cediranib risk: management of rotator cuff injury.

- Increased sample size from 132 to 138.

- Addition of a third dose reduction level for olaparib, $150 \mathrm{mg}$ two times per day.

- Reduction of CT scanning from every 8-12 weeks after 12 months of treatment.

- Addition of guidance on the use of concomitant novel oral anticoagulants.

\section{Consent or assent}

Potential participants were informed about the study and given a patient information sheet and allowed at least 24 hours to consider the study before the patients attended to give informed consent. Prior to giving consent, the trial was described by the investigator and patients were invited to ask any questions. Obtaining consent was delegated to suitably qualified and experienced investigators by the principal investigator at each site. The investigator is responsible for ensuring that the trial consent procedures comply with current applicable Good Clinical Practise (GCP) regulatory and ethical requirements.

By consenting into the trial, all participants will contribute to the translational research component. Patients were not eligible for the trial if they did not have sufficient archival tissue available.

\section{Confidentiality}

To protect the participants' privacy, the trial complies with the General Data Protection Regulation and Data Protection Act 2018. All data are anonymised. Date of birth and participant initials are collected for randomisation purposes in the randomisation database, which is kept separate from the case report form database and not used to identify participants. All documents will be stored securely and will only be accessible to study staff and authorised personnel.

\section{Access to data}

The sponsor will retain ownership of all data arising from the trial. Responsibility for data custodianship is delegated to OCTO. Explicit consent to retain and share anonymised data for use in future research will be obtained from all subjects. Requests for secondary use of the data after publication will be made in writing to OCTO and managed as per current applicable datasharing procedures.

\section{Ancillary and post-trial care}

Following the end of the final study visit (18 months post randomisation), participants can continue on the study treatment as long as there is clinical benefit. Only safety 
data will be collected after the 18 months of study visit. Participants who have progressed will be followed up for survival status at 12 and 18 months after randomisation or until death, whichever is sooner. All participants will have a treatment follow-up visit 28 days after treatment finishes.

\section{Dissemination plan}

The sponsor will retain ownership of all data arising from the trial. We aim to publish this research in a specialist peer-reviewed scientific journal on study completion. The results may also be reported at scientific meetings and/ or used for a thesis. The investigators will be involved in reviewing drafts of the manuscripts, abstracts, press releases and any other publications arising from the trial and will retain final editorial control.

Acknowledgements This study is conducted as part of the portfolio of trials in the registered UKCRC Oxford Clinical Trials Research Unit (OCTRU) at the University of Oxford. It follows their Standard Operating Procedures ensuring compliance with the principles of Good Clinical Practice and the Declaration of Helsinki and any applicable regulatory requirements. The authors acknowledge AstraZeneca provided support for this study and the NIHR for including this study in their portfolio. CRUK is also acknowledged as this trial was endorsed by Cancer Research UK [C1488/ A19909]. The contribution of IEPTOC members (Alastair Greystoke, Martin Forster, Janet Dunn, Shelley Petzer, Louise Carter, Melanie Costin, Tom Maishman, Stefan Symeonides), safety data reviewer (Sarah Blagden) and TMG (Stanley Kaye, lain McNeish, Rosalind Glasspool, Marcia Hall, Agnieszka Michael, Rene Roux) to the trial is acknowledged.The authors acknowledge the participants and sites involved. They also acknowledge English language editing by Dr Jennifer A. de Beyer, Centre for Statistics in Medicine, University of Oxford.

Contributors SN as the chief investigator was involved in the study's design and implementation. NM and AM are the trial manager, trial co-ordinator and trial statistician, respectively. RD and SD are the trial administrators current and previous. JH and LC provide statistical oversight and operational lead for the project, respectively. AM wrote the first draft of this manuscript, which all authors critically revised. All authors read and approved the final manuscript.

Funding This trial is funded by an educational grant from AstraZeneca (AZ) as part of the AZ-ECMC alliance. The trial is part of the NIHR portfolio. Any additional NHS clinical service support costs of patient care while on the study should be met by the host study site. The CRUK endorsement reference is C1488/A19909 and the label of project is ESR-14-10414. JH was supported by CRUK grant C49297/ A27294.

Competing interests None declared.

Patient consent for publication Not required.

Provenance and peer review Not commissioned; externally peer reviewed.

Supplemental material This content has been supplied by the author(s). It has not been vetted by BMJ Publishing Group Limited (BMJ) and may not have been peer-reviewed. Any opinions or recommendations discussed are solely those of the author(s) and are not endorsed by BMJ. BMJ disclaims all liability and responsibility arising from any reliance placed on the content. Where the content includes any translated material, BMJ does not warrant the accuracy and reliability of the translations (including but not limited to local regulations, clinical guidelines, terminology, drug names and drug dosages), and is not responsible for any error and/or omissions arising from translation and adaptation or otherwise.

Open access This is an open access article distributed in accordance with the Creative Commons Attribution Non Commercial (CC BY-NC 4.0) license, which permits others to distribute, remix, adapt, build upon this work non-commercially, and license their derivative works on different terms, provided the original work is properly cited, appropriate credit is given, any changes made indicated, and the use is non-commercial. See: http://creativecommons.org/licenses/by-nc/4.0/.

\section{ORCID iD}

Anita Mansouri http://orcid.org/0000-0002-7500-1636
REFERENCES

1 Bray F, Ferlay J, Soerjomataram I, et al. Global cancer statistics 2018: GLOBOCAN estimates of incidence and mortality worldwide for 36 cancers in 185 countries. CA Cancer J Clin 2018;68:394-424.

2 UK CR. Ovarian cancer incidence. Available: https://www. cancerresearchuk.org/health-professional/cancer-statistics/statisticsby-cancer-type/ovarian-cancer\#heading-Zero

3 Banerjee S, Kaye SB. New strategies in the treatment of ovarian cancer: current clinical perspectives and future potential. Clin Cancer Res 2013;19:961-8.

4 Markman M, Markman J, Webster K, et al. Duration of response to second-line, platinum-based chemotherapy for ovarian cancer: implications for patient management and clinical trial design. J Clin Oncol 2004;22:3120-5.

5 Bryant HE, Schultz N, Thomas HD, et al. Specific killing of BRCA2deficient tumours with inhibitors of poly(ADP-ribose) polymerase. Nature 2005;434:913-7.

6 Farmer H, McCabe N, Lord CJ, et al. Targeting the DNA repair defect in BRCA mutant cells as a therapeutic strategy. Nature 2005;434:917-21.

7 Fong PC, Boss DS, Yap TA, et al. Inhibition of Poly(ADP-Ribose) Polymerase in Tumors from BRCA Mutation Carriers. N Engl J Med Overseas Ed 2009;361:123-34.

8 Moore K, Colombo N, Scambia G, et al. Maintenance olaparib in patients with newly diagnosed advanced ovarian cancer. $N$ Engl J Med Overseas Ed 2018;379:2495-505.

9 Audeh MW, Carmichael J, Penson RT, et al. Oral poly(ADP-ribose) polymerase inhibitor olaparib in patients with BRCA1 or BRCA2 mutations and recurrent ovarian cancer: a proof-of-concept trial. The Lancet 2010;376:245-51.

10 Kaye SB, Lubinski J, Matulonis U, et al. Phase II, open-label, randomized, multicenter study comparing the efficacy and safety of olaparib, a poly (ADP-ribose) polymerase inhibitor, and pegylated liposomal doxorubicin in patients with BRCA1 or BRCA2 mutations and recurrent ovarian cancer. J Clin Oncol 2012;30:372-9.

11 Ledermann J, Harter P, Gourley C, et al. Olaparib maintenance therapy in patients with platinum-sensitive relapsed serous ovarian cancer: a preplanned retrospective analysis of outcomes by BRCA status in a randomised phase 2 trial. Lancet Oncol 2014;15:852-61.

12 Ledermann JA, Embleton AC, Raja F, Erratum: RF, et al. Cediranib in patients with relapsed platinum-sensitive ovarian cancer (ICON6): a randomised, double-blind, placebo-controlled phase 3 trial. Lancet 2016;387:1066-1074.

13 Liu JF, Barry WT, Birrer M, et al. Overall survival and updated progression-free survival outcomes in a randomized phase II study of combination cediranib and olaparib versus olaparib in relapsed platinum-sensitive ovarian cancer. Ann Oncol 2019;30:551-7.

14 Mirza MR, Åvall Lundqvist E, Birrer MJ, et al. Niraparib plus bevacizumab versus niraparib alone for platinum-sensitive recurrent ovarian cancer (NSGO-AVANOVA2/ENGOT-ov24): a randomised, phase 2, superiority trial. Lancet Oncol 2019;20:1409-19.

15 Perren TJ, Swart AM, Pfisterer J, et al. A phase 3 trial of bevacizumab in ovarian cancer. N Engl J Med 2011;365:2484-96.

16 Kaplan AR, Gueble SE, Liu Y, et al. Cediranib suppresses homologydirected DNA repair through down-regulation of BRCA1/2 and Rad51. Sci Transl Med 2019;11:eaav4508.

17 Belotti D, Vergani V, Drudis T, et al. The microtubule-affecting drug paclitaxel has antiangiogenic activity. Clin Cancer Res 1996;2:1843-9.

18 Kamat AA, Kim TJ, Landen CN, et al. Metronomic chemotherapy enhances the efficacy of antivascular therapy in ovarian cancer. Cancer Res 2007;67:281-8.

19 Lau D, Guo L, Gandara D, et al. Is inhibition of cancer angiogenesis and growth by paclitaxel schedule dependent? Anticancer Drugs 2004;15:871-5.

20 Lau DH, Xue L, Young LJ, et al. Paclitaxel (Taxol): an inhibitor of angiogenesis in a highly vascularized transgenic breast cancer. Cancer Biother Radiopharm 1999;14:31-6.

21 Milross CG, Mason KA, Hunter NR, et al. Relationship of mitotic arrest and apoptosis to antitumor effect of paclitaxel. J Natl Cancer Inst 1996;88:1308-14.

22 Tan DSP, Yap TA, Hutka M, et al. Implications of BRCA1 and BRCA2 mutations for the efficacy of paclitaxel monotherapy in advanced ovarian cancer. Eur J Cancer 2013;49:1246-53.

23 McNeish IA, Ledermann JA, Webber L, et al. A randomised, placebocontrolled trial of weekly paclitaxel and saracatinib (AZD0530) in platinum-resistant ovarian, fallopian tube or primary peritoneal cancer†. Ann Oncol 2014;25:1988-95.

24 Latimer NR, Abrams KR, Lambert PC, et al. Adjusting for treatment switching in randomised controlled trials-a simulation study 
